\title{
Robustness Evaluation Strategy of Ubiquitous Power Internet of Things Based on Important Node Recognition
}

\author{
Lu You ${ }^{1}$, Pan Zhiyuan ${ }^{1}$ and $\mathrm{Xu} \mathrm{Wei}^{1 *}$ \\ State Grid of China Technology College, Jinan, Shandong, 250002, China
}

\begin{abstract}
This paper analyses the structure and characteristics of ubiquitous power Internet of things (UPIoT) from four levels: the perception layer, network layer, platform layer and application layer. The robustness of UP-IoT is defined from the perspective of system structure, and the internal and external disturbance factors of robustness are analysed. According to the scale-free characteristics of complex network, a robustness evaluation strategy for UP-IoT based on identification of important nodes is proposed. A set of robustness evaluation indexes, including degree centrality, betweenness centrality, closeness centrality, maximum connectivity and connectivity factors, are established to quantify the importance of nodes. The model in this paper is used to analyse the UP-IoT network model with 12 nodes and verify the feasibility of the evaluation strategy.
\end{abstract}

\section{Introduction}

At present, the rapid development of economy and society has an increasing demand for energy, which not only puts forward higher requirements for the reliability of power supply, but also for the informatization and intelligence level of power production, power grid operation and maintenance, customer service, operation and management and other aspects. In order to adapt to the new situation, generate new drivers of growth, create new businesses and open up new markets, and seize opportunities and initiative in the new round of reform, power grid enterprises will build a smart service system. This system integrates advanced information technology with strong smart grid [1]. Big data, cloud computing, Internet of things, mobile Internet, artificial intelligence, block chain, edge computing and other technologies are widely used in the system. It has omni-directional state perception, efficient information processing, convenient and flexible application and other capabilities. It can realize the interconnection of everything, man-machine interaction and information sharing in the whole process of power production and every link of power system. This system is called "ubiquitous power Internet of things" [2]. The construction of UP-IoT will rely on the strong smart grid, and extend the current power communication network. After completion, the scale of network links, data processing capacity and the degree of business connection will be greatly improved, and the "three-stream integration" [3] of energy flow, business flow and data flow can be realized. It's a typical complex network [4]. The robustness [5] of complex networks has been widely concerned as a key indicator to evaluate the viability of the system. Therefore, it is necessary to design robust evaluation strategies according to the characteristics of UP-IoT, maintain real-time attention to network functions, and provide guidance for the construction, operation and control of UP-IoT.

\section{The structure and characteristics of UP-IOT}

\subsection{The structure of UP-IoT}




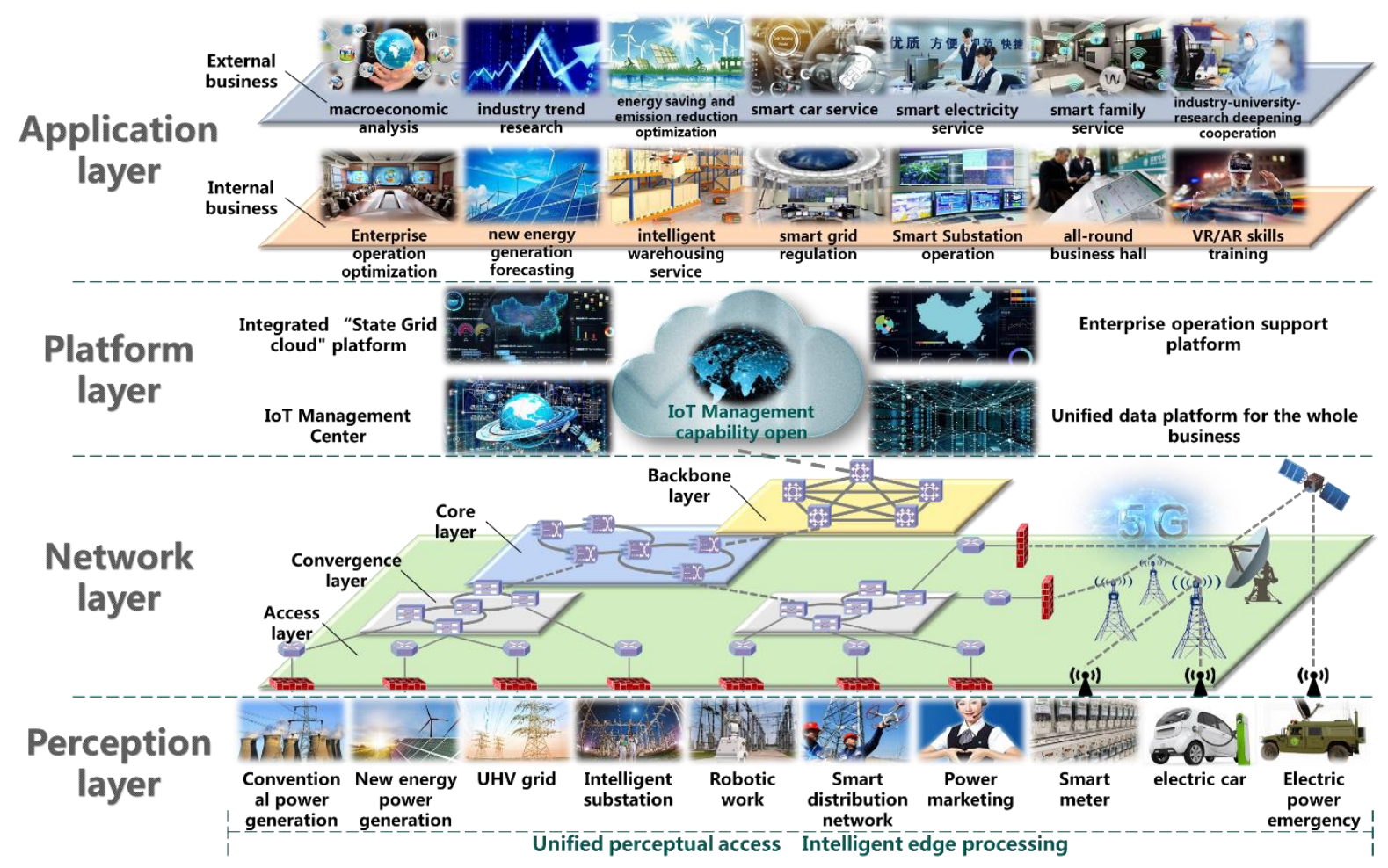

Figure 1. The structure of UP-IoT

Based on the strong smart grid and the concept of ubiquitous connectivity of things [6], it realizes the information connection and interaction of any time, any place, anyone and anything, which constitutes the UPIoT. The whole network is divided into four levels [7] according to function, that is, the perception layer, the network layer, the platform layer and the application layer. The structure of UP-IoT is shown in figure 1.

The perceptual layer is at the "edge" of the UP-IoT. It is equipped with various types of sensors, pattern recognition equipment and video monitoring equipment to extract real-time data and state information of the whole power production process and every link of the power system. It uses the related standardized communication protocol to connect the terminal communication node with the standardized communication interface and become the source of data interaction. The perception layer has the ability of edge intelligent processing [8]. It integrates access, communication, computing, storage and other functions to complete local processing of original data. It reduces network cloud computing pressure and transmission burden.

The network layer is the "vein" connecting cloud platform and edge nodes. It takes the power optical fiber communication network as the backbone, and the wireless communication network constructed by $5 \mathrm{G}$ [9] communication and satellite communication as the extension, and integrates the edge nodes in each scene into the communication network through the gateway equipment. According to its functions, the network layer can be further divided into access layer, convergence layer, core layer and backbone layer. Network bandwidth, transmission speed, number of nodes, and number of connections of a single node are successively increased.
The access layer has a large number of network nodes, which are widely distributed in various power plants, substations of $110 \mathrm{kV}$ and lower voltage grades, counties and their backup, power users, power emergency units and other scenarios, supporting data access and highspeed information interaction of local users; The network nodes in the convergence layer are mainly distributed in key scenarios such as $220 \mathrm{kV}$ substation, HVDC converter stations, regional power grid dispatching centers and backup dispatching centers, and the main functions are access control and flow control. The network nodes of the core layer mainly rely on the regional power grid dispatching centers, the provincial power grid dispatching center and its backup dispatching center, and the substation of voltage grade $330 \mathrm{kV}$ and above, with high transmission speed, low delay and high reliability. The network nodes of backbone layer are supported by national power grid dispatching center, regional power grid dispatching centers and provincial power grid dispatching centers to realize high-speed transmission and exchange of cross-regional power grid communication.

The platform layer can be regarded as the "background" of the UP-IoT. It is built based on big data and cloud computing technology. Its main role is to store and manage data of each scene, count and analyze information of each unit, calculate and verify indicators of each link, and provide a compatible and shared operation control environment and big data support for internal and external application services. The platform layer of the UP-IoT mainly includes four aspects: the integrated "state grid cloud" platform, the enterprise operation support platform, the IoT management center, and the unified data platform of the whole business. 
The application layer is the "front desk" of data and user interaction in the UP-IoT. It fully processes and excavates big data to make the potential value of data apparent, presents and interacts the corresponding indicators and information to users in an intuitive and friendly way, and meets the application needs of various types of users inside and outside the industry.

\subsection{The characteristics of UP-loT}

The original business system of smart grid is built based on the needs of each major. Although it is vertically connected, it is horizontally independent, and the crossmajor interaction and sharing of data and information is not ideal, forming a number of isolated business groups. The UP-IoT will unify data acquisition and interaction standards, standardize interface mode, realize horizontal connection of business and cross-professional sharing of data, and open the "last kilometre" of smart grid communication with wireless communication technology. It will expand the application space of data information with smart mobile terminal devices [10] with flexible access and friendly interface, and provide users with high-quality, efficient and simple services, so as to optimize the business operation mode and personnel cooperation mode. Therefore, the construction of UP-IoT will promote the emergence of new business forms and bring unprecedented opportunities for the development of power grid and enterprise transformation.

The UP-IoT integrates sensing, access, transmission, computing, storage and other equipment into various scenarios of power systems at all levels such as power plants, substations, dispatching centers, power users, etc. Each scene is connected by power network and communication network, forming an intelligent service system with interconnection of elements, state awareness, open sharing and integration innovation ability. These highly integrated scenarios of Internet of things devices become the elements that affect the function of the whole system. According to the complex network theory, the scene units integrating a large number of ubiquitous devices and facilities in the power Internet of things are abstracted as nodes, and the power network and communication network connecting each scene are abstracted as edges, so as to facilitate the application of complex network theory to study the UP-IoT.

The application of mobile Internet technology makes node access more convenient, networking more flexible and topology more complex. With the continuous access of new nodes, the scale of UP-IoT will continue to grow, and new access nodes tend to connect with nodes with high connectivity. A few nodes usually have a large number of connections, while most nodes have a small number of connections. That is to say, the connection degree of nodes conforms to power law distribution, which reflects the scale-free characteristics of complex networks [11].

\section{Robustness and disturbance factors analysis of UP-IoT}

\subsection{Robustness of UP-loT}

UP-IoT can be regarded as a generalized complex control system from the perspective of system theory. Among them, various levels and links, such as state perception, information extraction, edge calculation, signal access, data transmission and application processing, will be affected by complex physical environment and network environment, as well as interference from many known and unknown factors in the actual operation. System parameter perturbation is inevitable, so it is necessary to take robustness as an evaluation index of system performance and introduce it into UP-IoT.

The robustness of UP-IoT is mainly manifested in the ability to maintain stable network performance and normal operation of the system when the network suffers shock or parameter disturbance. Since the UP-IoT is divided into four levels from the structure, the disturbance can be decomposed according to the levels, so the robustness of the UP-IoT can be described as follows:

$R=\sum_{i=1}^{k_{1}} a_{i} Z_{i}+\sum_{i=1}^{k_{2}} b_{i} W_{i}+\sum_{i=1}^{k_{3}} c_{i} T_{i}+\sum_{i=1}^{k_{4}} d_{i} Y_{i}$

Among them, $R$ is the robustness of UP-IoT; $Z_{i}$ is the disturbance factor affecting the perception layer, $W_{i}$ is the disturbance factor affecting the network layer, $T_{i}$ is the disturbance factor affecting the platform layer, and $Y_{i}$ is the disturbance factor affecting the application layer. $a_{i}, b_{i}, c_{i}$ and $d_{i}$ are weights corresponding to different factors at different levels, $k_{1}, k_{2}, k_{3}$ and $k_{4}$ are the number of disturbance factors of each layer. This description mode reflects the system structure of UP-IoT on the whole, so the analysis of network structure is the key point to study the robustness of UP-IoT.

\subsection{Disturbance factors analysis}

According to the sources, the robustness disturbance factors of UP-IoT can be divided into internal factors and external factors. Internal factors are mainly the parameter perturbations of the system itself. After verification, the factors that have great influence include: signal acquisition success rate, sensor node density, sensor node failure rate, sensor node signal-to-noise ratio, signal acquisition throughput, signal transmission delay, delay jitter, terminal packet loss rate, terminal error rate, available bandwidth and working frequency band of the terminal, etc. [12]

External factors refer to impact damage, network attack, natural disaster, etc. Due to the small number of connections of most nodes, UP-IoT shows strong robustness in response to random attacks or unexpected failures. Due to the large number of connections of a few nodes, when the UP-IoT is maliciously attacked by these few nodes, once the node with a high number of connections fails, the network function will be severely hit, and even the network disintegration and paralysis 
will occur. It is of great significance to evaluate the robustness of network in real time for formulating network security strategies and doing network operation and maintenance well.

\section{Robustness evaluation strategy and evaluation indicator system}

\subsection{Robustness evaluation strategy}

According to the scale-free characteristic of complex networks, it can be seen that nodes with high connectivity are usually the key factors affecting network functions. Although these nodes have a small number, their importance is very high, which should be paid special attention to in network security protection and resource allocation. However, node connectivity is only one factor in evaluating network robustness. Therefore, the robustness evaluation strategy of UP-IoT is to establish a set of reasonable evaluation indicators, quantify the importance of network nodes by calculating the value of various indicators, determine the "key few" nodes that have a great impact on network functions from a macro perspective, and identify the key parts that determine the robustness of the network.

\subsection{Robustness evaluation indicator system}

The complex topological structure of UP-IoT can be abstracted into nodes and the edges of connecting nodes. The two nodes interact with each other through the connected edges and are not restricted by fixed directions and weights. According to these, the model of UP-IoT is constructed as undirected and unweighted [13] network $G=(V, E)$. The network is composed of $|V|=$ $n$ nodes and $|E|=m$ edges. $V$ is the set of nodes in the network, $V=\left\{v_{1}, v_{2}, v_{3}, \cdots, v_{n}\right\} . E$ is the set of edges in the network, $E=\left\{e_{1}, e_{2}, e_{3}, \cdots, e_{m}\right\}$.If node $v_{i}$ is connected to node $v_{j}$ via an edge, then $v_{i j}=1$. Conversely, if $v_{i j}=0$, then node $v_{i}$ is not connected to node $v_{j}$. Since the node cannot be connected to itself, $v_{i i}=0$ for any node $v_{i}$. The importance degree of $v_{i}$ of any node can be quantified as several key parameters of the node, including degree centrality [14], betweenness centrality [15], closeness centrality [16], maximum connectivity [17] and connectivity factor [18], etc. The importance degree of the node can be determined by accounting and analyzing the key parameters of each node.

First, degree centrality is an indicator describing the number of edges directly connected to a node. This indicator reflects that the more connections a node has, the greater its communication capacity and influence range will be, and the higher its importance will be. However, it is impossible to distinguish the importance of nodes with the same number of connections. The degree centrality of node $v_{i}$ is expressed as:

$$
D C(i)=\frac{1}{n} \sum_{j \neq i}^{n} v_{i j}
$$

Second, betweenness centrality is an indicator describing the number of shortest paths between one node and the other. This indicator reflects the influence of a node on the communication function of other nodes. If this indicator value of a node is high, it will have a greater impact on the network communication function and its importance will be higher. The shortest path number of all nodes in the network to other nodes is expressed as $g_{p q}$, and the number of all shortest paths passing through node $v_{i}$ is expressed as $g_{p q}(i)$, then the betweenness centrality of node $v_{i}$ is expressed as:

$$
B C(i)=\sum_{p \neq i \neq q}^{n} \frac{g_{p q}(i)}{g_{p q}}
$$

Third, closeness centrality is an indicator describing the inverse shortest path from a given node to other nodes. This indicator reflects the degree of closeness between nodes and other nodes in the network. If this indicator value of nodes is high, it is more likely that they are located in the center of the network and more important; otherwise, it indicates that nodes are marginalized by the network and have less influence. $d_{i j}$ represents the shortest distance between node $v_{i}$ and node $v_{j}$, then the closeness centrality of node $v_{i}$ is expressed as:

$$
C C(i)=n\left(\sum_{j \neq i}^{n} d_{i j}\right)^{-1}
$$

Fourth, the maximum connectivity $G_{\max }$ is the ratio of the number of nodes in the maximum connected subnets to the number of nodes in all connected subnetworks after the node is damaged and fails. The larger the $G_{\max }$ value is, the smaller the influence of the failure node on the network function, the stronger the robustness of the network, and the better the communication ability is maintained. When node $v_{i}$ is damaged, the node number of the maximum connected subnet is expressed as $N_{\max }$, and the node number of all connected subgraphs without isolated nodes is expressed as $N^{\prime}$, then the maximum connectivity of the network is expressed as:

$$
G_{\max }=N_{\max } / N^{\prime}
$$

Fifth, the connecting factor $\varpi$ is the reciprocal of the number of subnets formed by network splitting after the node is damaged and failed. $\varpi$ is larger, the smaller the impact on the function of network failure node, network fragmentation degree is lower, the stronger robustness of the network. When node $v_{i}$ is damaged, the network is divided into $\varepsilon$ sub-networks, and $\varepsilon$ contains isolated nodes. Then, the connectivity factor of the network is expressed as:

$$
\varpi=1 / \varepsilon
$$

\section{Establishment of network model and analysis of example}

\subsection{Establishment of network model}

In terms of topological structure, the UP-IoT is compatible with the typical topology of strong smart grid, and integrates classic structures such as star, tree, bus and ring. In order to ensure uninterrupted power supply 
and communication transmission and enhance network robustness, important nodes in the strong smart grid usually adopt ring networking mode to ensure $100 \%$ redundant backup of links. Structural features can be extracted and abstracted into network models, as shown in figure 2. The network model is composed of 12 nodes according to the typical topology of UP-IoT. Node 4, Node 5, Node 6 and Node 7 are in a group, and Node 6, Node 7 and Node 8 are in a group, respectively forming a ring network and the two rings are tangent. Node 1, Node 2, Node 3, Node 9, Node 10, Node 11 and Node 12 form star or tree links. It contains classical structures such as ring network, ring tangency and ring chain, etc. The maximum node degree is 4 , and the average node degree is 2.1667 .

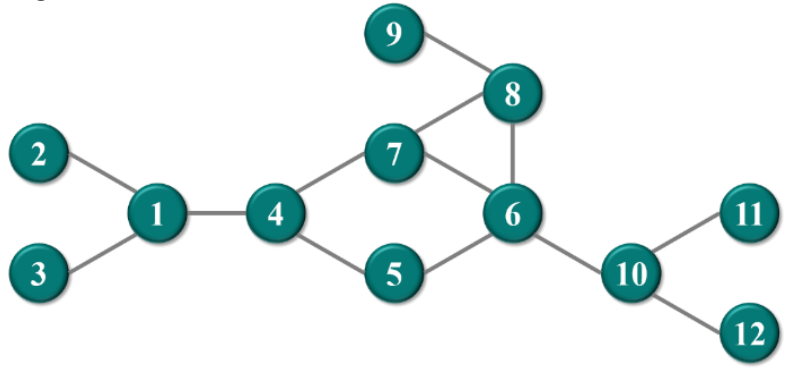

Figure 2. Network model

\subsection{Validation and analysis of evaluation strategies}

According to the robustness evaluation strategy, five evaluation indicators of each node in the network model are calculated one by one, including degree centrality DC, betweenness centrality $\mathrm{BC}$, closeness centrality $\mathrm{CC}$, maximum connectivity $G_{\max }$ and connectivity factor $\varpi$. The calculation results are shown in Table 1. According to the maximum connectivity of all nodes, $G_{\max }$ of Node 4 and Node 6 are the same and the minimum. Therefore, when Node 4 and Node 6 fail, the network functions are most affected, and the degree centrality value, betweenness centrality value and closeness centrality value of Node 6 are the highest, indicating that Node 6 is the most important in this network model. The connectivity factor $\varpi$ value of Node 1 and Node 10 is minimum because of star network mode. When Node 1 and Node 10 fail, Node 2, Node 3, Node 11 and Node 12 become isolated nodes, indicating that this connection mode is not conducive to maintaining network functions and reduces network robustness. The betweenness centrality value of Node 2, Node 3, Node 5, Node 9, Node 11 , and Node 12 is 0 , indicating that these nodes have no influence on the communication function of other nodes. Among them, the closeness centrality value of Node 2, Node 3, Node 9, Node 11, and Node 12 is low, indicating that they are located at the edge of the network. Node 5 has a high value of closeness centrality, indicating that it is close to the core of the network. Although Node 5 forms circular connections with Node 4, Node 6 and Node 7, it is not in the shortest path between any nodes, so the ring structure can improve the network robustness. From the above analysis, it can be seen that the importance of nodes cannot be distinguished from only one indicator. Through calculation and analysis of the network node 5 indicators, not only can determine the importance of nodes, but also can grasp about distribution of nodes in the network location, as well as the information such as networking mode and the influence of nodes on network functions. It can provide important reference for further targeted network construction and optimize the allocation of resources.

Table 1. 5 indicator values of each node in the network model

\begin{tabular}{cccccc}
\hline Node ID & $D C$ & $B C$ & $C C$ & $G_{\max }$ & $\varpi$ \\
\hline 1 & 0.25000 & 0.28788 & 0.38710 & 1.00000 & 0.33333 \\
2 & 0.08333 & 0.00000 & 0.29268 & 1.00000 & 0.50000 \\
3 & 0.08333 & 0.00000 & 0.29268 & 1.00000 & 0.50000 \\
4 & 0.25000 & 0.36364 & 0.48000 & 0.72727 & 0.50000 \\
5 & 0.16667 & 0.00000 & 0.48000 & 1.00000 & 0.50000 \\
6 & 0.33333 & 0.37879 & 0.52174 & 0.72727 & 0.50000 \\
7 & 0.25000 & 0.12121 & 0.52174 & 1.00000 & 0.50000 \\
8 & 0.25000 & 0.15156 & 0.46154 & 1.00000 & 0.50000 \\
9 & 0.08333 & 0.00000 & 0.33333 & 1.00000 & 0.50000 \\
10 & 0.25000 & 0.28788 & 0.41379 & 1.00000 & 0.33333 \\
11 & 0.08333 & 0.00000 & 0.30769 & 1.00000 & 0.50000 \\
12 & 0.08333 & 0.00000 & 0.30769 & 1.00000 & 0.50000 \\
\hline
\end{tabular}




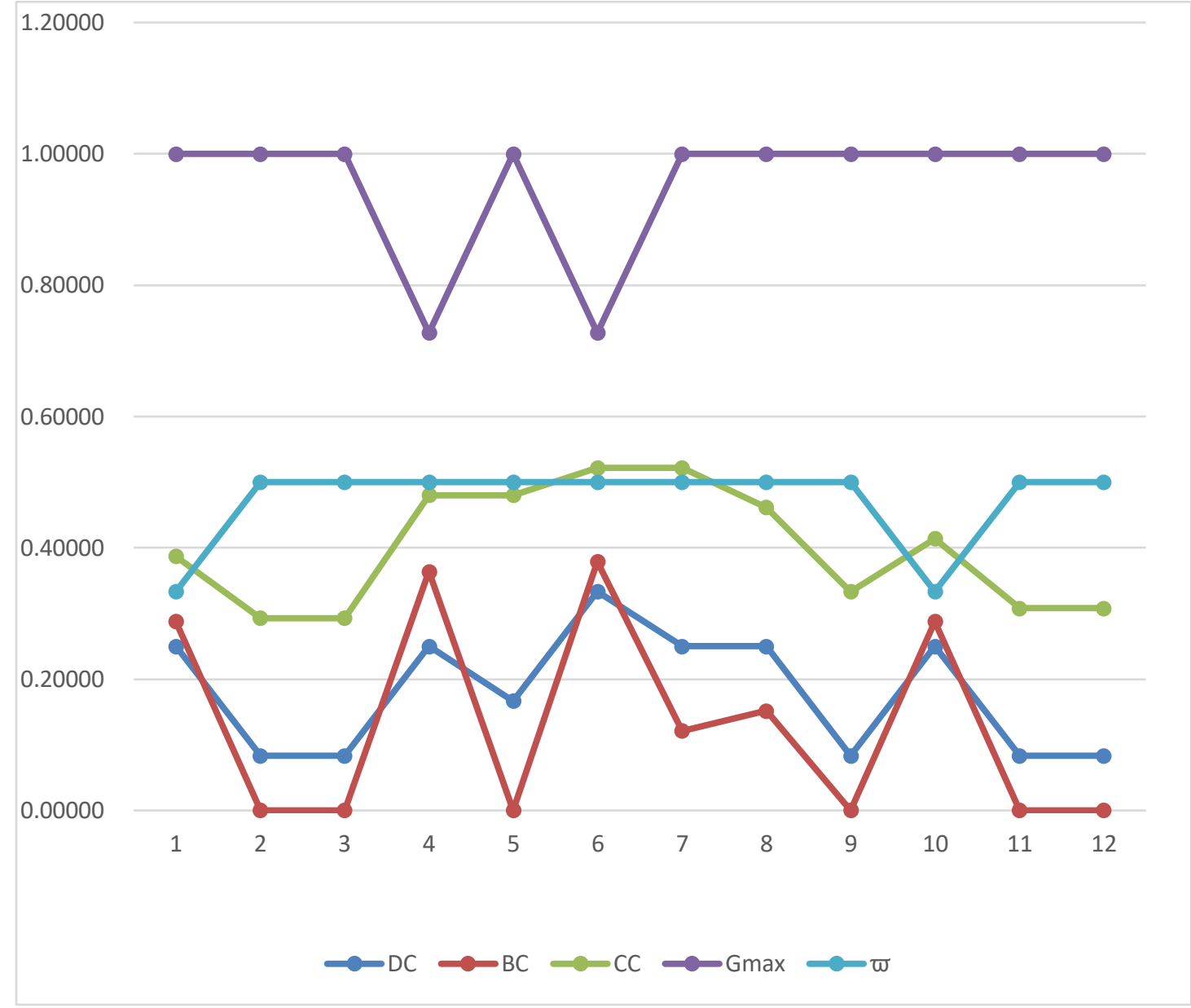

Figure 3. A polyline graph of the five indicator values of each node in the network model

The five indicator values of each node in the network model are drawn into a polyline graph, as shown in figure 3 . From the figure, we can intuitively see the change of the maximum connectivity of the network when a node fails. Then, the most important node in the network can be identified as node 6 by comparing other indicators. When Node 6 fails, the network robustness is the lowest. When some edge nodes, such as Node 2, Node 3, Node 9, Node 11 and Node 12, are attacked, the maximum connectivity and connectivity factor of the network are at high values, and the network has high robustness.

\section{Conclusion}

In this paper, a network model is constructed based on the typical UP-IoT topology, and the feasibility of the robustness evaluation strategy proposed in this paper is verified by an example analysis, which lays a foundation for the promotion and application in the actual network. UP-IoT has a huge scale. In the state of interconnection of everything, electrical equipment or substations can be abstracted as nodes in the network. Through state awareness, the real-time state of the network and each node can be obtained. It is necessary to give full play to the functions of cloud computing, locate important nodes immediately, identify the key factors affecting network functions, and realize real-time evaluation of network robustness, ensure the overall security and stability of the network, and improve the disaster tolerance and fault tolerance of UP-IoT.

\section{References}

1. Zhu Yi. Innovation and interconnection are the key to UP-IoT [N]. China electric power news,2019-0601(002).

2. Li Gang. Pioneer in the construction of pan-power Internet of things [N]. State grid report,2019-0404(003).

3. Zeng Ming, Wang Yuqing, Li Mingzhu, Dong Houqi, Zhang Xiaochun, Wang Haolei, Huo Xianxu, Zhang Zhigang. Preliminary study on pan-UP-IoT system architecture and implementation scheme [J]. Smart power,2019,47(04):1-7+58.

4. Sun ke. Application research of complex network theory in power network [D]. Zhejiang university,2008.

5. Chen Jun. Multi-layer vector Internet of things traffic network damage resistance and robustness analysis $[\mathrm{J}]$. Science and technology bulletin,2014,30(08):35-37.

6. Yang Dongsheng, Wang Daohao, Zhou Bowen, Chen Qiyu, Yang Zhile, Xu Guoyi, Cui Mingjian. Key technologies and application prospects of UP- 
IoT $[\mathrm{J}]$. Power generation technology,2019,40(02):107-114.

7. Chen Qiyu. Research on implementation strategy of UP-IoT [J]. Power generation technology,2019,40(02):99-106.

8. Li Kenli, Liu Chubo. Edge intelligence: current situation and prospect [J]. Big data,2019,5(03):69-75.

9. Shi Haozhen. Research on key technologies of $5 \mathrm{G}$ mobile communication network $[\mathrm{J}]$. Shandong industrial technology,2019(16):147.

10. Ou Xiatao. Research on intelligent warehouse of power grid companies -- based on Internet of things and mobile terminal technology $[\mathrm{J}]$. Economic research guide,2018(36):135-136.

11. Zhao Aoyang. Research on topology robustness optimization strategy of scale-free Internet of things [D]. Dalian university of technology,2017.

12. Ren Min. Robustness Evaluation Method Based on Cloud Model in Internet of Thing[D]. Taiyuan university of technology,2015.

13. Mo Hongming. Recognition method of node importance in complex network based on multiple attributes [J]. Journal of lanzhou university of arts and sciences (natural science edition), 2008,32(05):68-74.

14. Deng Wenhao,Zhang Bin,Zou Wenjin,Zhang Xiaofei,Cheng Xiongchao,Guan Lijie,Lin Yin,Lao Guohui,Ye Biyu,Li Xuan,Yang Chanjuan,Ning Yuping,Cao Liping. Abnormal Degree Centrality Associated With Cognitive Dysfunctions in Early Bipolar Disorder.[J]. Frontiers in psychiatry,2019,10.

15. Sunil Kumar R.,Kannan Balakrishnan. Betweenness centrality in Cartesian product of graphs[J]. AKCE International Journal of Graphs and Combinatorics, 2019.

16. Li Gaoshi,Li Min,Wang Jianxin,Li Yaohang,Pan Yi. United neighborhood closeness centrality and orthology for predicting essential proteins.[J]. IEEE/ACM transactions on computational biology and bioinformatics, 2018 .

17. Anonymous. ABB Ability MineOptimize Offers Maximum Connectivity[J]. Rock Products, 2019,122(4).

18. Behzad Ghanbarian,Marios A. Ioannidis,Allen G. Hunt. Theoretical Insight Into the Empirical Tortuosity - Connectivity Factor in the Burdine Brooks - Corey Water Relative Permeability Model[J]. Water Resources Research,2017,53(12). 